\title{
Clustering of Mycobacterium tuberculosis strains from foreign-born patients in Korea
}

\author{
Christie Y. Jeon, ${ }^{1,2} \uparrow$ HeeYoon $\mathrm{Kang}^{2}{ }^{2} \dagger$ Mihye $\mathrm{Kim}^{2}{ }^{2}$ Megan B. Murray, ${ }^{3,4}$ \\ Heejin Kim, ${ }^{2}$ Eun Hee Cho ${ }^{5}$ and Young Kil Park ${ }^{2}$ \\ ${ }^{1}$ Mailman School of Public Health, Columbia University, New York, NY, USA \\ ${ }^{2}$ Korean Institute of Tuberculosis, Chungcheongbuk-do, Republic of Korea \\ ${ }^{3}$ Harvard School of Public Health, Boston, MA, USA \\ ${ }^{4}$ Harvard Medical School, Boston, MA, USA \\ ${ }^{5}$ Korea Center for Disease Control and Prevention, Chungcheongbuk-do, Republic of Korea
}

Correspondence

Young Kil Park

ypark7@empal.com

Received 1 June 2011

Accepted 28 July 2011

\section{INTRODUCTION}

A substantial proportion of tuberculosis (TB) cases in Western Europe and North America occur among foreignborn individuals (Dahle et al., 2001; Iñigo et al., 2007; Long et al., 2002; Raviglione et al., 1993; Talbot et al., 2000). Concerns for potential impact of imported TB cases on national TB control have led to several molecular epidemiological studies examining the contribution of immigrant TB cases to recent transmission. Some studies attribute $\mathrm{TB}$ in foreigners to reactivation of latent infections and conclude that foreign $\mathrm{TB}$ cases do not contribute to extensive transmission in the host country (Borgdorff et al., 2000; Dahle et al., 2007). Other studies show that recent transmission likely plays an important role (Cohen \& Murray, 2005) and that there is active transmission between native and immigrant populations, fuelling the concern that increased immigration sets forth

†These authors contributed equally to this paper.

Abbreviations: DST, drug-susceptibility testing; KIT, Korean Institute of Tuberculosis; MDR, multidrug-resistant; MIRU-VNTR, mycobacterial interspersed repetitive unit-variable number of tandem repeat; $O R$, odds ratio; TB, tuberculosis; XDR, extensively drug-resistant.

A supplementary table is available with the online version of this paper. challenges for future TB control (Iñigo et al., 2007; Alonso Rodríguez et al., 2009; Zhou et al., 2008).

Migration patterns and TB control policies in Asia set a different context for spread and containment of TB compared to the Western setting. Korea presents a unique setting for studying the epidemiology of TB in migrants, as the number of registered foreign-born residents has been increasing rapidly, with a 4.8 -fold increase from 182788 in 1998 to 870636 in 2009 (Korean Statistical Information Service, 2010). While the burden of TB in Korea has drastically decreased since 1965 when TB prevalence was estimated at 668 per 100000 (Hong et al., 1998), TB incidence has stagnated at between 72 and 90/100 000 person-years since the year 2000 (WHO, 2010). In light of the recent trend, the Korean government has introduced a comprehensive plan to reduce the burden of TB through active screening in high-risk groups, establishing public-private mix strategies, strengthening TB surveillance, ensuring monitoring and evaluation of TB management, implementing measures for efficient contact tracing and expansion of treatment for latent TB infection. One of the prominent challenges to reducing the burden of $\mathrm{TB}$ will be the inadvertent importation of $\mathrm{TB}$ from countries of higher TB burden and higher prevalence of drug-resistant $\mathrm{TB}$, as well as circulation of $\mathrm{TB}$ in immigrant populations with poor access to health care. 
In order to better understand the potential impact of immigration on TB control in Korea, we conducted a retrospective molecular epidemiological study to investigate the transmission patterns and drug-resistance profiles of TB among foreign-born residents in Korea and to assess clustering of strains with Mycobacterium tuberculosis strains from native Koreans.

\section{METHODS}

Isolate selection. We selected clinical $M$. tuberculosis isolates archived at the Korean Institute of Tuberculosis (KIT), which routinely performs culture confirmation and drug-susceptibility testing (DST) on sputum samples referred by public health clinics in the National TB Program. In addition to the routine testing, KIT has been genotyping culture-confirmed $M$. tuberculosis samples from foreign-born residents since 2004. There were $1361 \mathrm{~TB}$ cases in foreign-born residents, including those who were diagnosed only by chest radiographs, reported to the Korean TB Surveillance System by public health clinics from 2004 to 2009. Of these, 397 were confirmed as $M$. tuberculosis infection by culture, and 306 culture-positive $M$. tuberculosis isolates were referred to the KIT for genotyping from 2004 to 2009. We report the results of $288 \mathrm{M}$. tuberculosis strains for which a sufficient amount of culture was available for genotyping and DST. We first assessed clustering among the immigrant TB genotypes to determine transmission events within the foreign-born populations. To investigate clustering with strains from native Koreans, we merged the genotype data from the foreign-born TB patients with a genotype database of 4780 culture-confirmed $M$. tuberculosis isolates from newly diagnosed native Korean TB patients under the age of 40 whose sputum was referred to the KIT by public health clinics from 2005 to 2009. Limited funding permitted genotyping only a portion of all isolates in the archive; thus, the KIT had decided a priori to genotype cultures only from younger TB patients in whom there is a greater expected extent of clustering.

Genotyping. M. tuberculosis isolates from both foreign-born patients and native Koreans were genotyped by IS6110 RFLP (Park et al., 2000). Briefly, chromosomal DNA was restricted with PvuII and digested DNA was separated by PFGE. Resulting DNA banding patterns were analysed by Bionumerics 5.1 (Applied Maths) and dendrograms were generated using the Dice coefficient and the unweighted pair group method with arithmetic averages. We defined clusters as those isolates with identical RFLP patterns. Clustered isolates from foreign-born residents with fewer than six DNA bands were also analysed by the standard 15-loci mycobacterial interspersed repetitive unit-variable number of tandem repeat (MIRU-VNTR) typing method for epidemiological discrimination (Supply et al., 2006). We further characterized all the strains as being $\mathrm{K}$ or $\mathrm{K}$ family strains by RFLP patterns as previously published (Kang et al., 2010; Kim et al., 2001; Park et al., 2000). K family strains were defined as strains with at least $80 \%$ similarity to the $\mathrm{K}$ strain that also have IS6110 DNA band copies in the range of eight to 12, five of which had to coincide at exactly the same location as in the standard K strain. All $\mathrm{K}$ and $\mathrm{K}$ family strains have been previously described to be within the Beijing family of strains (Kang et al., 2010). We ruled out the possibility of sample contamination by confirming that the date of the sample collection and culture of isolates with identical patterns were separated by more than 1 week.

DST. We summarized the DST results of M. tuberculosis isolates from foreign-born residents. Sputum samples sent by public health clinics were inoculated on Löwenstein-Jensen slants. They were tested for susceptibility to multiple drugs, using the agar proportion method, at the following concentrations: isoniazid, $0.2 \mu \mathrm{g} \mathrm{ml} \mathrm{g}^{-1}$; rifampicin, $40 \mu \mathrm{g} \mathrm{ml}^{-1}$; streptomycin, $10 \mu \mathrm{g} \mathrm{ml}^{-1}$; kanamycin, $40 \mu \mathrm{g} \mathrm{m}{ }^{-1}$; capreomycin, $40 \mu \mathrm{g} \mathrm{ml}^{-1}$; ofloxacin, $2 \mu \mathrm{g} \mathrm{ml}^{-1}$; moxifloxacin, $2 \mu \mathrm{g}$ $\mathrm{ml}^{-1}$; amikacin, $40 \mu \mathrm{g} \mathrm{ml}^{-1}$; levofloxacin, $2 \mu \mathrm{g} \mathrm{ml} \mathrm{m}^{-1}$. DST for pyrazinamide was done by pyrazinamidase test. Multidrug-resistant (MDR) TB was defined as TB resistant to at least isoniazid and rifampicin and extensively drug-resistant (XDR) TB was defined as MDR TB with additional resistance to ofloxacin and one of the three injectable second-line TB drugs capreomycin, kanamycin and amikacin.

Statistical analysis. We examined age, sex, previous TB treatment or nationality as risk factors for MDR TB or clustering by logistic regression. We performed multivariate regression with factors that were associated with the outcome at a significance level of $P<0.20$, and interpreted final odds ratios (OR) at the significance level of $P<0.05$.

\section{RESULTS}

We identified 288 TB cases in foreign-born patients reported through the public health clinics from 2004 to 2009 for which DST and genotype results were available. The largest numbers of isolates from foreign-born patients were reported in Seoul City $(43 \%)$ and the Gyeonggi province $(37 \%)$. Fifty-nine per cent of the population was male, and $62 \%$ was under 40 years of age. The majority were Chinese $(63.2 \%)$, while a substantial number came also from the Philippines (7.6\%), Mongolia (6.9\%) and Vietnam (6.3\%) (Table 1). Fifty-two isolates (18.1\%) were taken from patients who had received at least 1 month of TB treatment as indicated in the TB registry or by self report. Among those who were newly diagnosed, 23 (9.7\%) had MDR TB, and among those who had received TB treatment, $22(42 \%)$ had MDR TB. One previously treated patient had XDR TB (Table 2).

Table 1. Number of foreign-born TB patients by nationality

\begin{tabular}{|lc|}
\hline Country & No. of patients (\%) \\
\hline China & $182(63.2)$ \\
Philippines & $22(7.6)$ \\
Mongolia & $20(6.9)$ \\
Vietnam & $18(6.3)$ \\
Thailand & $7(2.4)$ \\
Indonesia & $6(2.1)$ \\
Pakistan & $5(1.7)$ \\
Russia & $4(1.4)$ \\
Bangladesh & $3(1.0)$ \\
Nepal & $3(1.0)$ \\
Taiwan & $3(1.0)$ \\
Cambodia & $1(0.3)$ \\
Ethiopia & $1(0.3)$ \\
Kazakhstan & $1(0.3)$ \\
Myanmar & $1(0.3)$ \\
Ukraine & $1(0.3)$ \\
Uzbekistan & $1(0.3)$ \\
Unknown & $9(3.1)$ \\
\hline
\end{tabular}


Table 2. Drug-resistance profile of $M$. tuberculosis strains from foreign-born TB patients in South Korea

\begin{tabular}{|lcc|}
\hline Drug resistance & $\begin{array}{c}\text { No. (\%) of patients among newly } \\
\text { diagnosed }(\boldsymbol{n}=\mathbf{2 3 6})\end{array}$ & $\begin{array}{c}\text { No. (\%) of patients among } \\
\text { previously treated }(\boldsymbol{n}=\mathbf{5 2})\end{array}$ \\
\hline Isoniazid (non-MDR) & $33(14.0)$ & $10(19.2)$ \\
Rifampicin (non-MDR) & $3(1.3)$ & $1(1.9)$ \\
Streptomycin (non-MDR) & $26(11.0)$ & $6(11.5)$ \\
Ofloxacin (non-MDR) & $1(0.4)$ & $0(0.0)$ \\
MDR & $23(9.7)$ & $22(42.3)$ \\
MDR with resistance to ofloxacin but not injectable drug & $2(0.8)$ & $3(5.8)$ \\
MDR with resistance to injectable drug but not ofloxacin & $0(0.0)$ & $2(3.8)$ \\
XDR & $0(0.0)$ & $1(1.9)$ \\
\hline
\end{tabular}

Previous TB treatment and Chinese nationality were independently associated with $\mathrm{MDR} \mathrm{TB}$ in univariate $\left(\mathrm{OR}_{\text {Previous treatment }}=6.8,95 \% \mathrm{CI} 3.4-14 ; \mathrm{OR}_{\mathrm{China}}=4.5,95 \%\right.$ CI 1.9-11) and multivariate (adjusted $\mathrm{OR}_{\text {Previous treatment }}=$ 5.1, $95 \%$ CI 2.4-11; adjusted $\mathrm{OR}_{\text {China }}=3.0,95 \%$ CI 1.1-9.3) analysis (Table 3 ). Among TB patients from China, $13 \%$ (17/ 135) of newly diagnosed and $47 \%$ (25/47) of previously treated patients had MDR TB.

When we assessed clustering by RFLP patterns among 288 TB cases in foreign-born patients only, we identified 38 isolates from foreign-born individuals that were not unique, six of which had fewer than six DNA bands and were subsequently genotyped by MIRU-VNTR typing (M31, M32). After excluding four strains with unique MIRU-VNTR profiles (Supplementary Table S1 in JMM Online), we identified $34(12 \%)$ strains that formed 14 clusters. Of these, 21 strains were part of 10 clusters that included only isolates from patients from China. The other four clusters involved transmission events across different nationalities, indicating recent cross-cultural transmission in the host country. For example, cluster M18 involved patients from China, Russia and Uzbekistan (Table 4).

When we combined the IS6110 RFLP genotype data of the M. tuberculosis isolates from foreign-born cases with those from the native Korean population, we identified a total of $51(17.7 \%)$ M. tuberculosis isolates from foreign-born individuals that formed 31 clusters, including the 14 clusters of strains from foreign-born cases identified above, as well as 17 additional clusters not previously identified (Table 4, RFLP patterns available upon request).

Strains from a number of foreign-born patients belonged to RLFP clusters comprising $\mathrm{K}$ or the $\mathrm{K}$ family subtype of M. tuberculosis strains, which have been found to occur endemically in Korea (Kim et al., 2001; Park et al., 2000) (Table 4). Of note, clusters M1 and M3 were particularly large, mostly comprising strains from native Korean TB cases, with a few strains from cases from China, Mongolia and Taiwan. Other clusters were composed of strains that did not belong to the $\mathrm{K}$ family subtypes, and contained relatively fewer strains from cases among native Koreans.

Table 3. Predictors of MDR TB and clustering of TB subtypes among foreign-born TB patients in South Korea

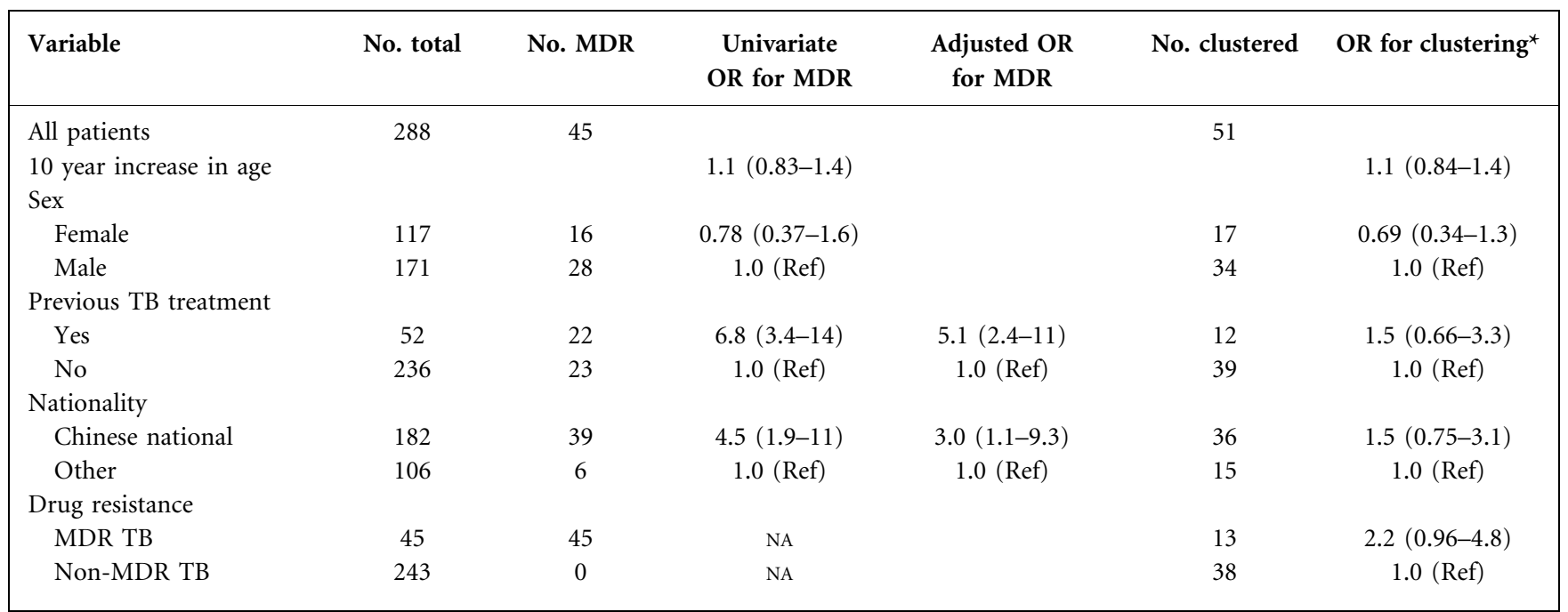

NA, Not applicable; (Ref), reference group.

${ }^{\star}$ Multivariate analysis not performed as only one factor was associated with clustering at $P<0.20$. 
Table 4. Clustering of $M$. tuberculosis strains from foreign-born TB patients in South Korea

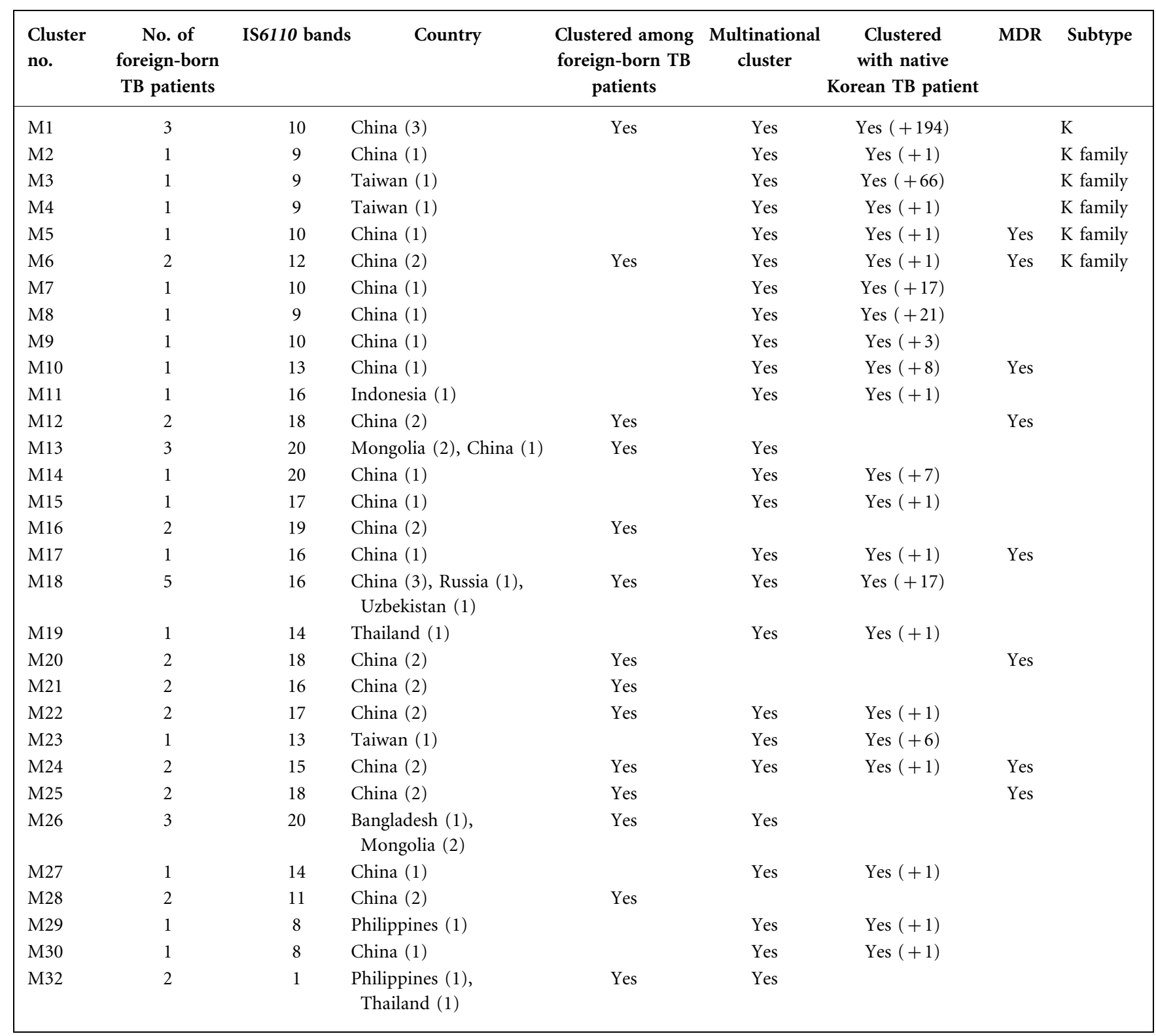

Eight clusters among cases in foreign-born patients contained an MDR TB strain, all of which were isolated from patients from China. DST results of clustered strains from native Koreans were not available for this study (Table 4).

In a univariate analysis, we found that age, sex, previous TB treatment, Chinese nationality and MDR status were not associated with clustering (Table 3 ).

\section{DISCUSSION}

Contrary to the notion that TB disease among immigrants results from reactivation of a previous infection (Borgdorff et al., 2000; Dahle et al., 2007), we found that active transmission may be occurring among different foreign-born population groups and between foreignborn individuals and the native Koreans, as evidenced by multinational clusters. Interestingly, we suspect that in some incidences, native Korean TB patients may be the source of infection for foreign-born TB patients, as $\mathrm{K}$ family strains endemic in Korea (Kim et al., 2001; Park et al., 2000) were identified in foreign-born TB patients. A previous study in Norway also found that outbreaks of TB involving foreign-born individuals were often determined to be of Norwegian origin (Dahle et al., 2003). The origin of strains uncommon in Korea was less clear; some strains, especially those with high RFLP copy numbers, resembled a modern Beijing family type that may have potential for global transmission as they have been identified in multiple countries surrounding Korea (Kang et al., 2010). 
We found a clustering rate of $17.7 \%$ among strains from foreign-born TB patients referred to the KIT by public health clinics that are widely distributed throughout South Korea, thus providing national sampling. The clustering rate is similar to that found in previous molecular epidemiology conducted in Japan $(5 / 32,15.6 \%$ ) (Ohkado et al., 2008) and Hong Kong (21/110, 19\%) (Chan-Yeung et al., 2006). However, the clustering rate is likely underestimated given that public health clinics manage only $20-30 \%$ of the TB population in Korea, the rest being managed by private clinics with variable follow-up and reporting rates.

MDR TB was highly prevalent among the foreign-born patients, especially among those from China. The prevalence figures were comparable to the highest reported estimates of MDR TB prevalence in China (10.4\% in newly diagnosed patients in Liaoning, $37 \%$ in previously treated patients in Inner Mongolia) (He et al., 2008). This contrasts with the reported MDR TB prevalence in South Korea: $2.7 \%$ among newly diagnosed TB patients and $14 \%$ among previously treated TB patients (WHO, 2010). Similar to our findings, drug-resistant $\mathrm{TB}$ has been reported to occur more frequently in TB patients from China who reside in British Columbia (Moniruzzaman et al., 2006), Hong Kong (Law et al., 2008) and Australia (Lumb et al., 2006). The high prevalence of MDR TB in people from China is of concern given that people from China make up the greatest number of foreigners in Korea, many of whom are actually ethnically Korean, such as descendants of Korean nationals who had migrated out of Korea during a period of Japanese occupation and the Korean War. Being ethnically Korean, such 'foreigners' have few language and cultural barriers, allowing them to assimilate into Korean society. Thus, opportunities for transmission to and from native Koreans may be greatest for these individuals. This may explain the relatively higher clustering rate among those with MDR TB, although the association between MDR and clustering was not significant. A previous study in Spain also found that TB transmission occurs more often between groups who speak the same language (Alonso Rodríguez et al., 2009).

Our study was limited by a number of factors. First, while the foreign-born population is the largest studied in Asia, the sample comprised only those patients referred by the public sector, which is estimated to treat $20-30 \%$ of the TB patients in Korea. This may have led to an underestimate of the clustering rate. Data from the private sector were not available because laboratory diagnostics and TB notification have not been uniformly employed in this sector. However, the geographical distribution of the genotyped isolates and of total notified TB patients in Korea showed no noticeable difference, indicating representativeness of the included isolates. Also, MIRU-VNTR typing results were not available for strains from native Koreans; thus, we could not determine clustering with native Koreans for strains with fewer than six RFLP bands. The employment of newer methods, such as MIRU-VNTR typing, would allow further discrimination of strains in clusters determined by IS6110. While genotyping by IS6110 is useful for discriminating strains, its results are often limited in portability and challenging to compare across laboratories (Mathema et al., 2006). Considering also the fact that $M$. tuberculosis strains in certain countries in Asia (i.e. Vietnam and the Philippines) often present fewer than six IS6110 bands, future research should consider the use of genotyping techniques, such as MIRU-VNTR typing, that would be optimal for discriminating and comparing strains across different settings. Further, details on the date of entry into Korea and contact tracing were lacking, thus we were not able to determine the temporal and spatial context of the transmission. Nonetheless, the use of molecular epidemiological data provides information on transmission events that would not be available from standard contact tracing (Borrell et al., 2009). In addition, records on previous $\mathrm{TB}$ treatment outside Korea were determined by self-report; thus, previously treated TB cases may have been misclassified as newly diagnosed, biasing the prevalence of primary and acquired MDR TB.

In conclusion, we found that while the clustering rate was not high, the clusters showed the potential for active crosscultural transmission of TB among foreign-born individuals, with possible transmission to and from the native Korean community. Further, we determined that MDR TB prevalence was high among foreign-born $\mathrm{TB}$ patients referred to public sector clinics. In light of the findings from our study, we recommend a larger molecular epidemiological study in the greater Asian context to investigate the extent of international transmission of $\mathrm{TB}$, particularly of MDR forms.

\section{ACKNOWLEDGEMENTS}

This work was supported by the J. William Fulbright Foreign Scholarship Board and the Harvard University Asia Center, and data were provided by Korean Institute of Tuberculosis and Korea Center for Disease Control.

\section{REFERENCES}

Alonso Rodríguez, N., Chaves, F., Iñigo, J., Bouza, E., García de Viedma, D., Andrés, S., Cías, R., Daza, R., Domingo, D. \& other authors (2009). Transmission permeability of tuberculosis involving immigrants, revealed by a multicentre analysis of clusters. Clin Microbiol Infect 15, 435-442.

Borgdorff, M. W., Behr, M. A., Nagelkerke, N. J., Hopewell, P. C. \& Small, P. M. (2000). Transmission of tuberculosis in San Francisco and its association with immigration and ethnicity. Int J Tuberc Lung Dis 4, 287-294.

Borrell, S., Español, M., Orcau, A., Tudó, G., March, F., Caylà, J. A., Jansà, J. M., Alcaide, F., Martín-Casabona, N. \& other authors (2009). Factors associated with differences between conventional contact tracing and molecular epidemiology in study of tuberculosis transmission and analysis in the city of Barcelona, Spain. J Clin Microbiol 47, 198-204. 
Chan-Yeung, M., Kam, K. M., Leung, C. C., Wang, J., Yew, W. W., Lam, C. W. \& Tam, C. M. (2006). Population-based prospective molecular and conventional epidemiological study of tuberculosis in Hong Kong. Respirology 11, 442-448.

Cohen, T. \& Murray, M. (2005). Incident tuberculosis among recent US immigrants and exogenous reinfection. Emerg Infect Dis 11, 725-728.

Dahle, U. R., Sandven, P., Heldal, E. \& Caugant, D. A. (2001). Molecular epidemiology of Mycobacterium tuberculosis in Norway. J Clin Microbiol 39, 1802-1807.

Dahle, U. R., Sandven, P., Heldal, E. \& Caugant, D. A. (2003). Continued low rates of transmission of Mycobacterium tuberculosis in Norway. J Clin Microbiol 41, 2968-2973.

Dahle, U. R., Eldholm, V., Winje, B. A., Mannsåker, T. \& Heldal, E. (2007). Impact of immigration on the molecular epidemiology of Mycobacterium tuberculosis in a low-incidence country. Am J Respir Crit Care Med 176, 930-935.

He, G. X., Zhao, Y. L., Jiang, G. L., Liu, Y. H., Xia, H., Wang, S. F., Wang, L. X., Borgdorff, M. W., van der Werf, M. J. \& van den Hof, S. (2008). Prevalence of tuberculosis drug resistance in 10 provinces of China. BMC Infect Dis 8, 166.

Hong, Y. P., Kim, S. J., Lew, W. J., Lee, E. K. \& Han, Y. C. (1998). The seventh nationwide tuberculosis prevalence survey in Korea, 1995. Int J Tuberc Lung Dis 2, 27-36.

Iñigo, J., García de Viedma, D., Arce, A., Palenque, E., Alonso Rodríguez, N., Rodríguez, E., Ruiz Serrano, M. J., Andrés, S., Bouza, E. \& Chaves, F. (2007). Analysis of changes in recent tuberculosis transmission patterns after a sharp increase in immigration. $J$ Clin Microbiol 45, 63-69.

Kang, H. Y., Wada, T., Iwamoto, T., Maeda, S., Murase, Y., Kato, S., Kim, H. J. \& Park, Y. K. (2010). Phylogeographical particularity of the Mycobacterium tuberculosis Beijing family in South Korea based on international comparison with surrounding countries. $J \mathrm{Med}$ Microbiol 59, 1191-1197.

Kim, S. J., Bai, G. H., Lee, H., Kim, H. J., Lew, W. J., Park, Y. K. \& Kim, Y. (2001). Transmission of Mycobacterium tuberculosis among high school students in Korea. Int J Tuberc Lung Dis 5, 824-830.

Korean Statistical Information Service (2010). Number of Registered Foreigners. Daejeon: Statistics Korea.

Law, W. S., Yew, W. W., Chiu Leung, C., Kam, K. M., Tam, C. M., Chan, C. K. \& Leung, C. C. (2008). Risk factors for multidrug-resistant tuberculosis in Hong Kong. Int J Tuberc Lung Dis 12, 1065-1070.

Long, R., Sutherland, K., Kunimoto, D., Cowie, R. \& Manfreda, J. (2002). The epidemiology of tuberculosis among foreign-born persons in Alberta, Canada, 1989-1998: identification of high risk groups. Int J Tuberc Lung Dis 6, 615-621.

Lumb, R., Bastian, I., Crighton, T., Gilpin, C., Haverkort, F. \& Sievers, A. (2006). Tuberculosis in Australia: bacteriologically confirmed cases and drug resistance, 2004: a report of the Australian Mycobacterium Reference Laboratory Network. Commun Dis Intell 30, 102-108.

Mathema, B., Kurepina, N. E., Bifani, P. J. \& Kreiswirth, B. N. (2006). Molecular epidemiology of tuberculosis: current insights. Clin Microbiol Rev 19, 658-685.

Moniruzzaman, A., Elwood, R. K., Schulzer, M. \& FitzGerald, J. M. (2006). Impact of country of origin on drug-resistant tuberculosis among foreign-born persons in British Columbia. Int J Tuberc Lung Dis 10, 844-850.

Ohkado, A., Nagamine, M., Murase, Y., Uchimura, K., Kaguraoka, S., Tatsumi, Y., Yamada, N., Ohmori, M., Maeda, S. \& other authors (2008). Molecular epidemiology of Mycobacterium tuberculosis in an urban area in Japan, 2002-2006. Int J Tuberc Lung Dis 12, 548-554.

Park, Y. K., Bai, G. H. \& Kim, S. J. (2000). Restriction fragment length polymorphism analysis of Mycobacterium tuberculosis isolated from countries in the western pacific region. J Clin Microbiol 38, 191-197.

Raviglione, M. C., Sudre, P., Rieder, H. L., Spinaci, S. \& Kochi, A. (1993). Secular trends of tuberculosis in western Europe. Bull World Health Organ 71, 297-306.

Supply, P., Allix, C., Lesjean, S., Cardoso-Oelemann, M., RüschGerdes, S., Willery, E., Savine, E., de Haas, P., van Deutekom, H. \& other authors (2006). Proposal for standardization of optimized mycobacterial interspersed repetitive unit-variable-number tandem repeat typing of Mycobacterium tuberculosis. J Clin Microbiol 44, 4498-4510.

Talbot, E. A., Moore, M., McCray, E. \& Binkin, N. J. (2000). Tuberculosis among foreign-born persons in the United States, 1993-1998. JAMA 284, 2894-2900.

WHO (2010). Tuberculosis Country Profiles: Republic of Korea. Geneva: World Health Organization.

Zhou, Y., Khan, K., Feng, Z. \& Wu, J. (2008). Projection of tuberculosis incidence with increasing immigration trends. J Theor Biol 254, 215-228. 